\title{
ANVISA: an introduction to a new regulatory agency with many challenges
}

Kim Huynh-Ba ${ }^{1 *}$ and Alexandra Beumer Sassi ${ }^{2,3}$

\begin{abstract}
Brazil, the largest country in South America, has become the second largest pharmaceutical market in the emerging world. The Brazillian Health Surveillance Agency (Agencia Nacional de Vigilancia Sanitaria - ANVISA) was created in 1999 with the primary goal to protect and promote public health surveillance over products and services in Brazil. The governing body and structure of this new regulatory agency will be the aims of this paper, where the authors hope to share their understanding on the regulatory processes and its significant importance of this agency.
\end{abstract}

Keywords: Brazil, ANVISA, Regulatory, Registration, Resolution, Health surveillance, Public health

\section{Introduction}

Brazil is the largest country in South America with a population of over 200 million people (Fig. 1). As an emerging market, Brazil has become the second largest pharmaceutical market in the emerging world, with an expectation of economic growth between 7 to $10 \%$ annually until 2020 (Afonso et al. 2015). Global bio/ pharmaceutical companies are highly interested in investing in this vast and growing market. However, this opportunity may present a significant challenge when navigating through the complex Brazilian regulatory process.

\section{Brazilian agency: ANVISA (www.anvisa.gov.br)}

The Brazilian Health Surveillance Agency, commonly known as ANVISA, abbreviated from Portuguese "Agencia Nacional de Vigilancia Sanitaria," is the food and drug regulatory agency in Brazil. ANVISA was created in 1999 and is linked to the Ministry of Health. It is characterized by its administrative independence, financial autonomy, and the stability of its directors. In the federal public regulatory structure, the agency is connected to the Ministry of Health. ANVISA's primary goal is to protect and promote public health, by exercising health surveillance over products and services, including processes, ingredients, and technologies that pose any health risks.

* Correspondence: kim.huynhba@pharmalytik.com

${ }^{1}$ Pharmalytik LLC, Newark, DE, USA

Full list of author information is available at the end of the article
ANVISA's vision is to achieve legitimation in society as an integral part of the Brazilian Unified Health System, via a nimble, modern, transparent, and domestic and international benchmark in health surveillance and regulation. ANVISA's mission is "to protect and promote public health and to intervene in the risks caused by the production and use of products regulated by health surveillance. This mission must be carried out in coordination with states, municipalities and the Federal District, according to the Brazilian Unified Health System principles, to improve the quality of life of the population."

The agency is also responsible for health control in ports, airports, and borders, as well as for establishing relations with the Ministry of International Affairs and with foreign organisms and institutions to deal with international affairs regarding health surveillance.

ANVISA was accepted as a new regulatory member of the International Council on Harmonisation of Technical Requirements for Registration of Pharmaceuticals for Human Use (ICH). As part of the objective to extend its global outreach, ICH, in November 2016, welcomed ANVISA from Brazil and the Ministry of Food and Drug Safety (MFDS) from South Korea as the first new regulatory Members, together with the Biotechnology Innovation Organization (BIO) as a new industry association Member. There are now 13 members and 22 observers.

\section{Responsibilities}

ANVISA is responsible for drug registration and licensure of pharmaceutical laboratories and other companies 


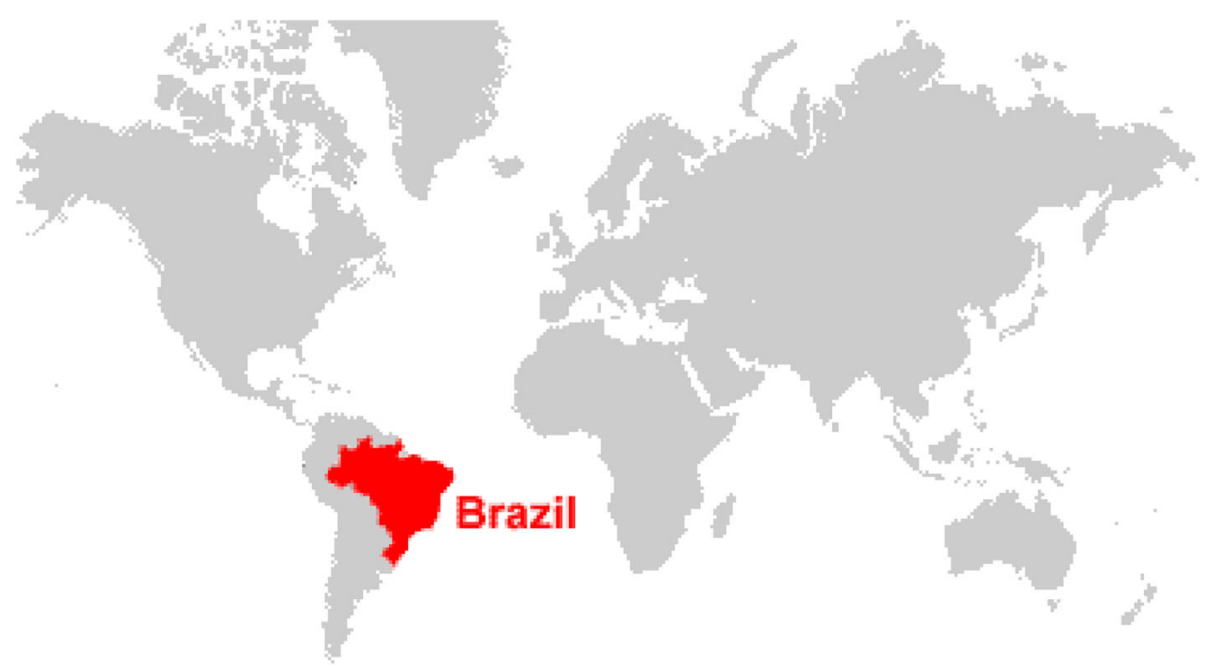

Fig. 1 Location of Brazil. https://wiredre.com/wp-content/themes/new042911/images/brazil-map.gif

inside the pharmaceutical production flow. The agency is also responsible for establishing regulations applicable to clinical trials (with regards to drugs Chemistry, Manufacturing, and Control (CMC) and subject safety). In conjunction with the Health Ministry and other ministries members, ANVISA works with the Chamber of Drug Market Regulation (CMED) to regulate drug pricing. Ethical human clinical trials are in turn regulated by an Ethics Committee (EC) linked to the Health Ministry. ANVISA controls a broad diversity of health-related areas, as shown in Table 1.

Together with states and local municipalities, the agency inspects factories, monitors the quality of drugs, exercises post-marketing surveillance, takes pharmacovigilance actions, and regulates drug promotion and marketing. Moreover, ANVISA evaluates patent requests related to pharmaceutical processes and products, in partnership with the National Industrial Property Institute (INPI).

ANVISA's values encompass ethics and responsibility as a public agency, the capacity for interaction and

Table 1 Areas regulated by ANVISA

- Blood and blood products

- Coordination of Brazillian Health Surveillance System

- Cosmetics

- Drugs

- Generic drugs

- Food

- Health services (e.g., hospitals)

- Drug Price regulations (part of a Committee)

- Medical devices

- Pharmacovigilance

- Ports, airports, and borders (health-related issues and products)

- REBLAS - Brazilian network of analytical laboratory

- Sanitizing products

- Tobacco

- Pesticides (also regulated by environmental agency IBAMA and by Ministry of Agriculture and Livestock) integration, management excellence, knowledge as a source of action, transparency, and accountability.

\section{Regulatory submission process}

Companies need to understand the regulatory structure as well as their requirements to initiate product development in a new country. For the past five years, ANVISA has updated their regulations and developed numerous guidances. The primary challenge to successfully submitting an ANVISA regulatory file is directly associated with bio/pharmaceutical companies' lack of knowledge of the process. Currently, ANVISA's website is presented in two languages: Portuguese (the native language in Brazil) and English. However, the English version is limited in contents and does not contain all the relevant information that is presented in the Portuguese version. Companies that do not have a regulatory presence in Brazil may be dependent on regulatory professionals with specific expertise in that market.

To facilitate the better understanding of the ANVISA process, the registration procedure is compared to a more familiar registration system used by the U.S. Food and Drug Administration (FDA).

Table 2 summarizes the registration for each clinical phase and marketing authorization. All the submissions to ANVISA should be in the country's native language, Portuguese.

\section{How to change your CTD to a Brazilian registration?}

More and more, bio/pharmaceutical companies that currently file in large markets in the developed world such as The European Union, United States, and Japan are developing global regulatory strategies to reach other regions. The implementation of the Common Technical Document 
Table 2 Registration process of ANVISA vs. FDA

\begin{tabular}{|c|c|}
\hline ANVISA & FDA \\
\hline $\begin{array}{l}\text { 1. Pre-registration } \\
\text { a. Protocol for clinical study }\end{array}$ & Investigational New Drug (IND) \\
\hline $\begin{array}{l}\text { 2. Registration } \\
\text { a. Documents to be submitted } \\
\text { b. Protocol for the new drug } \\
\text { c. Protocol for import of the new drug }\end{array}$ & $\begin{array}{l}\text { New Drug Application (NDA)/ } \\
\text { Biologics License Application } \\
\text { (BLA) }\end{array}$ \\
\hline $\begin{array}{l}\text { 3. Post-registration } \\
\text { a. Change in registration, renewal } \\
\text { of registration } \\
\text { b. History of Product Change (HMP) }\end{array}$ & $\begin{array}{l}\text { a. Supplementary NDA/BLA } \\
\text { b. Annual Report NDA/BLA }\end{array}$ \\
\hline
\end{tabular}

(CTD) format facilitating the submission and review process for the countries that adopted the format established by the International Conference on Harmonization (ICH) is very common; however, for the countries that are new and have not adopted the CTD format, some changes are required from a CTD to the specific countries' regulations.

In the case of the Brazilian registration, there are regional specificities and similarities with the CTD format applicable in the ICH countries members. The Brazilian regulation shows that the dossier is structured for product registration in two main parts:

1. Administrative: Compilation of all administrative data, including specific requirements for imported products.

2. Technical: Technical reports, including quality, nonclinical, and clinical information, presenting similarities to the CTD Modules 2, 3, 4, and 5.

In November 2016, Brazil has officially become an ICH member, and the organization has been working diligently to prioritize and update their resolution to adapt ICH guidelines, including M4: Organization of the Common Technical Document for the Registration of Pharmaceuticals for Human Use. More activities are expected in this area as the harmonization process is being worked on.

\section{Conducting a clinical trial in brazil}

Brazil has become a large market and more attractive to the pharmaceutical industry, thus the desire to entering this market became more evident. It is helpful to know the regulatory institutions that are involved with the clinical trials conducted in Brazil and to be awared of related documents.

\section{Institutions involved in the approval process}

In Brazil, three different institutions: CONEP (Central), CEP (local committee), and ANVISA, are responsible for reviewing and approving regulatory documents to initiate a clinical study in this region. The CONEP and ANVISA processes happen in parallel.

1. Comissão Nacional de Ética em Pesquisa (CONEP), translated from Portuguese as National Committee of Ethics in Research: this committee is the Central Ethics Committee, which is related to the Ministry of Health, is responsible for review and approval of the ethical aspects of a clinical trial in Brazil.

a. The Coordinating CEP is responsible for submission of the clinical trial to the CONEP, and will directly work with the CONEP regarding the study assessment. A written letter will be issued if the CONEP approves the study.

b. Otherwise, CONEP will communicate questions to the Coordinating site through the Coordinating CEP (see bullet 2). Questions are raised in one of three options:

- Questions were raised: This is the case that the Coordinating CEP receives a set of questions from CONEP and forwards to the Coordinating Site and the Sponsor. The Sponsor will prepare the responses and comments on a question-by-question basis and send back to the Coordinating Site.

- Questions are raised under "approval with the recommendation": In this case, the clinical team must provide CONEP recommendations, along with all necessary updated documents to all sites for submission to the CEPS. All CEPs must review and approve the changes before the study starts at the respective sites.

- Questions are raised under "Pending" status: In this case, the clinical team must provide the recommendations, along with all necessary updated documents to the Coordinating Site only, for submission to the Coordinating CEP. After approval, the coordinating CEP submits the responses to CONEP for review. After CONEP approval of the study, all remaining sites and the respective CEPS must receive the updated approved documents for review and local approval before the study starts at each site.

2. Comitê de Ética em Pesquisa (CEP), translated from Portuguese as Committee of Ethics in Research: these are local ethics committees that are registered with CONEP (the central committee). They can review and approve clinical trials conducted at an institution.

a. Each company should have a list of documents and information required for the clinical trial. 
b. These documents and information are received from the sponsor and the study sites. These documents may contain templates of forms, documents, and statements required for the study. They also include statements related to general study information. For the first three study sites, one of these sites is selected as Coordinating Site; then its CEP is designated as the Coordinating CEP. All documentation must be provided in Portuguese; any translated document must be accompanied by the corresponding certificate of translation.

3. Agência Nacional de Vigilância Sanitária (ANVISA): this is the Brazilian Regulatory Agency. This group is responsible for reviewing all technical aspects and issuing the Import License for a clinical trial. Two types of dossiers are reviewed by ANVISA:

a. Processo de Anuência (Consent Process): the main application dossier for initial submission. This dossier will receive a unique specific number from ANVISA to be used for all updates.

b. Processo de Inclusão de Centros (Inclusion Centers Process): the dossier for adding sites when every site other than the one submitted in the Processo de Anuência. This document will carry the same number as the one previously assigned.

\section{Documentation required}

ANVISA prefers to have all information submitted entirely in the dossiers. However, it is acceptable to submit the dossier with part of the information pending and then update the remaining documents as they are available. Responses will be made to address each question that ANVISA raises.

Several documents submitted are briefly described below:

a. Brazilian Informed Consent Form (ICF) template that must be translated into Portuguese and adapted to the local government. There are three different types of ICF that one may find:

- Master ICF is the primary document that is submitted

- Revised ICF is done whenever the changes affect any local requirement.

- Revisions to site-specific ICF is any revision to a site-specific ICF.

b. Importation which includes documents related to Import License request. This document consists of any updates to the list of materials to be imported. It consists of all study drug documentation (such as Certificate of Analysis, BSE Certificates, and documents). This request is valid during one year of the clinical trial and multiple shipments. If the length of the clinical trial or quantities needed exceeds the approved terms, then an extension to cover the extra needs must be done.

c. All master labels for study drugs and materials to ensure they contain all the expected information. These labels are in the English language, and also in its translation to Portuguese.

d. Any advertisement or announcement for the study that will be distributed to the sites.

e. Data protection requirements to be met in the confidentiality and data handling section of the ICF.

f. Insurance and Indemnity assessed as the Sponsor must provide a Medical Assistance Letter for injuries related to the study.

g. Amendments included to the protocol. These amendments needed to be approved by each CEP before the implementation and notified to ANVISA. CONEP approval is not mandatory but can be requested.

h. Safety report must be done according to local requirements. This document is received from the Sponsor in both English and Portuguese.

i. Progress and annual reports are prepared and submitted following local requirements. The study staff must submit an interim report to its CEP every six months.

j. End-of-trial notification is similar to the Progress and Annual Reports at sites and with ANVISA.

k. Clinical trial reports are prepared and submitted to ANVISA only as required by the Sponsor.

\section{Approval process}

The regulatory approval process typically passes through 2 ethical evaluations: the institutional CEP and CONEP. The Coordinating Ethics Committee (EC) submits the multicenter research center research protocol for CONEP for review, and approval would apply to all sites. CONEP submission requires the protocol, the ICF, investigator's brochure, Power of Attorney, protocol approved a letter from Coordinating EC, and all other documents submitted to the Coordinating EC.

ANVISA has separate departments for drugs and devices: COPEC is the Coordination of Clinical Research with Drugs, and Biological Products and COPEA is the Coordination of Clinical Research with Devices and Food. ANVISA evaluates the clinical dossier of drug development rather than each trial. For individual clinical protocol, a simple submission package must be submitted when phase I, II or III trials in Brazil is to be performed; however, for phase IV, only a notification to ANVISA is required. According to their guidance, it could take from 90 to 180 days for ANVISA to review the dossier before the study can be initiated (Reuters 2014). 


\section{Recent updated resolutions}

In Brazil, it is required that all Active Pharmaceutical Ingredients (APIs) and drug products manufactured or imported are registered with the agency. The regulatory system though established but yet is quite complex. The drug registration is valid for five years and may be revalidated for equal and successive periods of time.

Their regulatory requirements and guidelines are written in the format called "resolution". As a new ICH member, ANVISA strives to revise many of their resolutions and bring them up-to-date, especially those topics that have corresponding ICH guidelines. However, many of these updated guidelines contain higher level of details with concrete structure, thus making it difficult to embrace the risk-based approached as compared to the original ICH countries. Below are some examples of recent distributed resolutions.

\section{Validation of analytical methods}

Resolution number 166, dated July 24th, 2017 establishes the criteria for the validation of analytical methods and other provisions. Similarly to other resolutions from ANVISA, the non-fulfillment of any criterion shall be technically justified and will be subjected to analysis by ANVISA. Resolution 166 brings clarity on validation parameters for methods used for pharmaceutical ingredients, drug products, and biological products in all production stages filling an essential gap in guidances for the sponsors. The scope of this resolution does not include microbiological methods that are compendial or have been technically justified.

The main point in this resolution is that an analytical method that is not described in the official compendium recognized by ANVISA requires an analytical validation. A full validation should include accuracy, repeatability precision, intermediate precision, selectivity, limit of detection, limit of quantification, linearity, and interval. Compendial analytical methods shall have their suitability for the intended use shown by a partial validation study. A partial validation should include at least the parameters of precision, accuracy, and selectivity.

In case of transfer of methods that have already been approved by ANVISA, a copy of the approved validation report or the petition number under which the final version of such report was filed must be provided. A revalidation of an analytical method will be considered when there are:

- Changes in the synthesis or obtainment of Active Pharmaceutical Ingredient (API);

- Changes in product composition;

- Changes in the analytical method;

- Other changes that may significantly impact the validated method.

\section{Forced degradation study (FDS) resolution}

Resolution RDC-53/15 introduced in 2015 has provided specific requirements to conduct forced degradation studies for product registration and post-approval changes. It outlines the reporting, identification, and qualification of degradation product beyond the $\mathrm{ICH}$ recommendations (Tattersall et al. 2016). A guideline was also issued about this topic (Guia 04/2015). In essence, this resolution requests that:

- Safety studies are necessary for degradation products if they are above a qualification limit

- Assessment of specific forced degradation conditions is completed and documented in a report.

- Consideration should be given to degradation resulted from manufacturing and storage.

- Mass balance should be obtained and explained.

This resolution also requests that the forced degradation studies should be reported in a report with specific requirements. The issue concerns many companies because ANVISA requires that this resolution will be applied not only to the new drug but also for some post-approval change submissions. These requirements would incur extensive work for the pharma industry to support approved products, which a large body of historical data may be available.

\section{Stability resolution}

Resolution RE No 1/RDC No 45 permits shelf life estimation to 24 months based on extrapolation of accelerated studies and 12 months long term. The expiration dating must be confirmed with actual 24 months long term studies. Although Brazil is now an ICH official member, ANVISA requires $\mathrm{WHO}$ Zone IVb for stability storage conditions, including Active Pharmaceutical Ingredient (API) that has different storage requirements based on the country of manufacture and exported to Brazil. It is also clear that both accelerated studies and long-term stability studies must be conducted. Unlike other ICH countries, Brazil required actual long term data for any shelf life beyond 24 months.

The follow-up stability studies are required for products imported in bulk or immediate packages and they must be conducted in Brazil. Also, a list of analytical tests is also included for different pharmaceutical dosage forms, thus it becomes mandatory for approval leaving less flexibility to be determined based on the drug products (ANVISA Resolution RE 2005; ANVISA Resolution RDC 2012; Nagao et al. n.d.). Bracketing and matrixing concepts, similar to Q1D, are presented in the annex with specific schedules indicating that ANVISA considers reduced testing. A direct comparison between ICH Q1A (R2) and ANVISA requirements for registration of inhalation 
products was discussed to show that there are still potential areas for harmonization that would benefit global drug development (Nagao et al. n.d.).

In Brazil, stability nonconformities represent a significant number of registration refusal causes, and in 2015, $11 \%$ of those were related to the absence of stability indicating methods during stability studies. This lack of discussion and effective instructions in Brazil regulation until 2015 may contribute to the drug product refusal registration (Moraes do Carmo et al. 2017). Brazilian requirements for stability indicating methods were compared with international guidelines such as $\mathrm{ICH}$, World Health Organization (WHO), and European Medicine Agency (EMA). At present, the stability resolution is being developed and published for public comments. With many specific requirements, it seems that the Brazilian requirements are more stringent than international ones. Thus opportunities for harmonization continue.

\section{Conclusions}

As a new organization, ANVISA has made critical strides to bring up the quality of product registration in Brazil. The new resolutions from ANVISA contains more detailed information and instructions, of which we think is very valuable. Unfortunately, it gives less flexibility to apply risk-based quality management concept. In a few cases, it even causes additional unnecessary testing without appropriate level of reasons. We also found that ANVISA has increased their hiring significantly in the past few years, thus training of new officers could be a challenge to keep up with monitoring and implementing the new changes. As this organization now is a member of ICH organization, one would expect more harmonization activities with $\mathrm{ICH}$ and also globally to find sufficient balance to ensure adequate data to support the quality of the products distributed in Brazil.

\section{Acknowledgments}

The authors gratefully acknowledge Raphael Sanches Pereira at ANVISA, Monica Whitmire at Catalent, and Oksana Golycheva at Johnson \& Johnson for reviewing this article and sharing their expert opinions.

\section{Funding}

The paper received a publication waiver from AAPS Open.

\section{Availability of data and materials}

The information and material presented are summaries of the referenced materials.

\section{Authors' contributions}

$\mathrm{KCH}$ and $\mathrm{ABS}$ made an equal contribution as the primary authors of this article. Both authors read and approved the final manuscript.

\section{Competing interests}

Both authors declare that they are employee or owner of the companies provided and therefore received payments from said companies. Otherwise, both authors declare that they have no competing financial interests.

\section{Publisher's Note}

Springer Nature remains neutral with regard to jurisdictional claims in published maps and institutional affiliations.

\section{Author details}

${ }^{1}$ Pharmalytik LLC, Newark, DE, USA. Voisin Consulting Life Sciences, Cambridge, MA, USA. ${ }^{3}$ Cardinal Health Regulatory Sciences, 7400 West 110 th St, Suite 300, Overland Park, KS 66210, USA.

Received: 20 August 2018 Accepted: 12 November 2018

Published online: 12 December 2018

\section{References}

Afonso P. Dreszer J., Francies T., Ramos P. Maintaining momentum in Brazil's pharmaceutical market. http://www.mckinsey.com/insights/health_systems_ and_services/maintaining_momentum_in_brazils_pharmaceutical_market. 2015

ANVISA Resolution RDC No 45, regulates the conductance of studies on active pharmaceutical ingredients stability, 9, 2012

ANVISA Resolution RE (2005) No 1, guide for the undertaking of stability. Studies Moraes do Carmo AC, Pereira RS, Gratieri T (2017) Brazilian requirements for stability indicating methods, science direct, trends in analytical chemistry. Elsevier

Nagao L., et al., n.d. A comparison of Brazil ANVISA and ICH Stability Requirements for Inhalation Products, https://ipacrs.org, https://ipacrs.org/ assets/uploads/outputs/Brazil_poster.pdf

Reuters T (2014) Reformatting Your CTD Information for Your Brazilian Registration Dossier.http://citeseerx.ist.psu.edu/viewdoc/download; jsessionid=BAB8867A2ED27A92D789E35CE7EB491B?doi=10.1.1.676. 2134\&rep=rep $1 \&$ type $=$ pdf

Tattersall P et al (2016) Impact from the recent issuance of ANVISA resolution RDC-53/2015 on pharmaceutical small molecule forced degradation study requirements. American Pharmaceutical Review 31 https://www.american pharmaceuticalreview.com/Featured-Articles/184364-Impact-from-the-RecentIssuance-of-ANVISA-Resolution-RDC-53-2015-on-Pharmaceutical-SmallMolecule-Forced-Degradation-Study-Requirements/

\section{Submit your manuscript to a SpringerOpen ${ }^{\circ}$ journal and benefit from:}

- Convenient online submission

- Rigorous peer review

- Open access: articles freely available online

- High visibility within the field

- Retaining the copyright to your article

Submit your next manuscript at $\boldsymbol{\nabla}$ springeropen.com 